\section{Short term insomnia}

Helga Peter

Marburg, Deutschland

\section{Synonyme}

Kurzdauernde Insomnie

\section{Definition}

In dem Bestreben, \ „Insomnien“ über ihre Dauer zu definieren, wurde 1983 durch die National Institutes of Health (NIH) die Bezeichnung short term insomnia eingeführt. Sie charakterisierte eine weniger als drei Wochen andauernde Insomnie. In der Praxis hatte sich der Begriff zunächst nicht bewährt. In den Versionen der ICSD (1990) und ICSD-2 (2005) erfolgte die Benennung unter ätiologischen Gesichtspunkten als Anpassungsbedingte Schlafstörung bzw. Schlafanpassungsstörung (Akute Insomnie). Die \ „ICSD-3“ stellt wieder den Aspekt der Dauer in den Vordergrund und benennt sie D „Kurzzeit-Insomnie“" (short term insomnia disorder).

Siehe auch $\triangleright$,Diagnostische Klassifikationssysteme“. 